\title{
A new model for early diagnosis of Alzheimer's disease based on BAT-SVM classifier
}

\author{
Shereen A. Taie ${ }^{1}$, Wafaa Ghonaim ${ }^{2}$ \\ ${ }^{1}$ Computer Science Department, Faculty of Computers and Information, Fayoum University, Egypt \\ ${ }^{2}$ Faculty of Science, Al-Azhar University, Cairo, Egypt
}

\section{Article Info \\ Article history: \\ Received Oct 11, 2020 \\ Revised Jan 28, 2021 \\ Accepted Feb 16, 2021}

\section{Keywords:}

Alzheimer's disease

BAT optimizer

Magnetic resonance imaging

hippocampus

Support vector machine

\begin{abstract}
Magnetic resonance images (MRI) of the brain is a significant tool to diagnosis Alzheimer's disease, for this reason we use it to measure regional changes in the brain that reflect disease progression to detect early stages of the disease. In this paper, we propose a new model that adopts Bat for parameter optimization problem of support vector machine (SVM) to diagnose Alzheimer's disease via MRI biomedical image. The proposed model uses MRI for biomedical image classification to diagnose three classes; normal controls (NC), mild cognitive impairment (MCI), and Alzheimer's disease (AD). The proposed model based on segmentation for the most involved areas in the disease hippocampus, the features of MRI brain images are extracted to build feature vector of the brain, then extracting the most significant features in neuroimaging to reduce the high dimensional space of MRI images to lower dimensional subspace, and submitted to machine learning classification technique. Moreover, the model is applied on different datasets for efficiency validation, it concluded that the new BatSVM model achieved acceptable level of accuracy reached to $95.36 \%$ using maximum number of bats equal to 50 and number of generation equal to 10 .
\end{abstract}

This is an open access article under the CC BY-SA license.

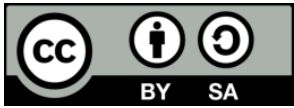

\section{Corresponding Author:}

Shereen A. Taie

Department of Computer Science

Faculty of Computers and Information

Fayoum University, Egypt

Email1: sat00@fayoum.edu.eg

\section{INTRODUCTION}

Alzheimer disease is considered as an evolution neurodegenerative disease, the conditions that result in the loss of memory and cognitive abilities which represent a public health problem. Early diagnosis is important to slow down neuro degeneration and providing optimal care, in particular for the mild cognitive impairment (MCI) patients which have a very high risk to develop AD in the near future [1]. There are many biomedical imaging tools to early diagnose $\mathrm{AD}$, such as magnetic resonance imaging (MRI) that help to detect and follow process of the brain atrophy evolution $[2,3]$. The value of MRI as an objective diagnostic tool for $\mathrm{AD}$ depends on the degree of AD detection by classification methods. Structural MRI identified local volumetric changes in brain areas with $\mathrm{AD}$ and MCI that associated longitudinal changes and cross-sectional differences in volume and size of particular brain regions, such as the hippocampus [4]. The most common prominent change in the brain structure for $\mathrm{AD}$ is the reduction of the volume of the hippocampus that recognized as an early feature of the degenerative process involved in AD [5]. Many researchers diagnose $\mathrm{AD}$ from the hippocampus region of interest (ROI) using extracted features [6-8]. Early memory decline is correlated to the appearance of reductions in hippocampus volume that is sensitive to the first stage of AD [9]. 
Numerous MRI techniques were proposed as non-invasive imaging biomarkers for the early diagnosis of $\mathrm{AD}$ and evaluation of disease progression. These biomarkers were analyzed to study of differences between groups, such as disease and healthy groups. Though, these biomarkers methods can not improve the clinical potential diagnosis due to these are not applicable on a single-subject level. To overcome these limitations machine-learning techniques were promising tools in data analysis of neuro imaging for individual class prediction. In AD classification and MCI prediction, many supervised classifiers have been used. For multivariate classification SVM are commonly used in AD classification [10]. Structural MRI recognized that in a specific brain regions $\mathrm{AD}$ and $\mathrm{MCI}$ associated longitudinal changes in volume and size such as the hippocampus and entorhinal cortex [11]. More recently, MRI data have a brain imaging powerful analysis tool which is machine learning techniques that aimed to classify AD vs. cognitively normal (NC) or MCI vs. NC [4].

The contribution of this paper is that it introduced a new model that build a categorized power and improve diagnostic decisions of AD using Bat based SVM classification for NC, MCI and AD subjects. The proposed new model compromises of four subsequent phases, segmentation phase to segment the most areas that involved disease hippocampus. Hence in the feature extraction and reduction phase the features of MRI brain images are extracted to build feature vector of the brain, where13 statistical features are extracted, then extracting the most significant features in neuroimaging to minimize MRI images dimensional space of to lower dimensional subspace. Moreover, in the classification phase the most significant reduced features were submitted to machine learning classification technique SVM with maximum accuracy $82 \%$. Finally, a new Bat-SVM model was presented for optimizing the parameter of the problem of the SVM to maximize the classification accuracy reached to $95.36 \%$.

\section{PROPOSED MODEL}

We have developed a new model that based on machine-learning techniques in data analysis of neuro imaging for individual class prediction to overcome the limitations of biomarkers methods which can not improve the clinical potential diagnosis. Machine-learning techniques aimed to classify AD vs. cognitively normal (NC) or MCI vs. NC through MRI data for the brain images. The innovative aspect of the proposed model is to build a categorized power and improve diagnostic decisions of AD using Bat based SVM classification for NC, MCI and AD subjects. K-fold strategy stratified cross validation used for enhancing generalization of SVM. We use 7-fold cross validation of measuring the model performance. As the primary randomly sample set was divided into $\mathrm{k}$ folds, one fold is used as test and for training it used the remaining k-1 folds. The proposed model comprises of four phases as illustrated in Figure 1 and discussed in details in the following subsections segmentation phase, feature extraction and reduction phase, classification phase and a new Bat-SVM phase.

\subsection{Segmentation phase}

The first phase is segmentation for the most disease areas in the hippocampus [12]. Medical image segmentation is intricate as a result of the various images characteristics. Image segmentation using thresholding techniques is a wildly applied in many applications and in medical images for simplicity and efficiency reasons. There are two main categories for the thresholding techniques, global thresholding is the first one in which a single threshold for all image pixels is used and the second one is the adaptive thresholding techniques that used various threshold values for different local areas [13]. In this paper Otsu's method [13] based global image threshold was used science the components pixel values and that of the background are both fully consistent in their respective values over the whole image. As illustrated in Figure 1, the segmented image contains hippocampus ROI.

\subsection{Feature extraction and reduction phase}

In the second phase, the features of MRI brain images are extracted to build feature vector of the brain. Discrete wavelet transform is an efficient tool for feature extraction, it allows analysis of images at various levels of resolution due to its multi-resolution analytic property [14]. Moreover, in the second phase the size of hippocampus ROI is calculated for both left side $(\mathrm{mmL})$, right side $(\mathrm{mmR})$ and utilized as one of the most significant extracted features, then 13 statistical features are extracted using the 2-D wavelet transform to build feature vector by the brain (contrast, correlation, energy, homogeneity, mean, standard deviation, entropy, RMS, variance, smoothness, kurtosis, skewness, IDM, size of mmL, size of mmR, and region size mean). However, discrete wavelet transform is computationally expensive so that feature selection is used to select the best discriminant biomarkers based on wavelet features and reduce the contained information of the MRI images. Principal component analysis (PCA) was used to reduce the dimensions of features [15]. PCA is efficient tool for extracting the most significant features; it is usually 
used in neuroimaging to reduce the high dimensional space of MRI images to lower dimensional subspace $[16,17]$. Focusing on local features extraction from ROIs known to be involved in AD. The proposed model first employed wavelet transform to extract features from images, followed by applying PCA to reduce the dimensions of features. The reduced features were submitted to SVM.

Bat SVM Optimization

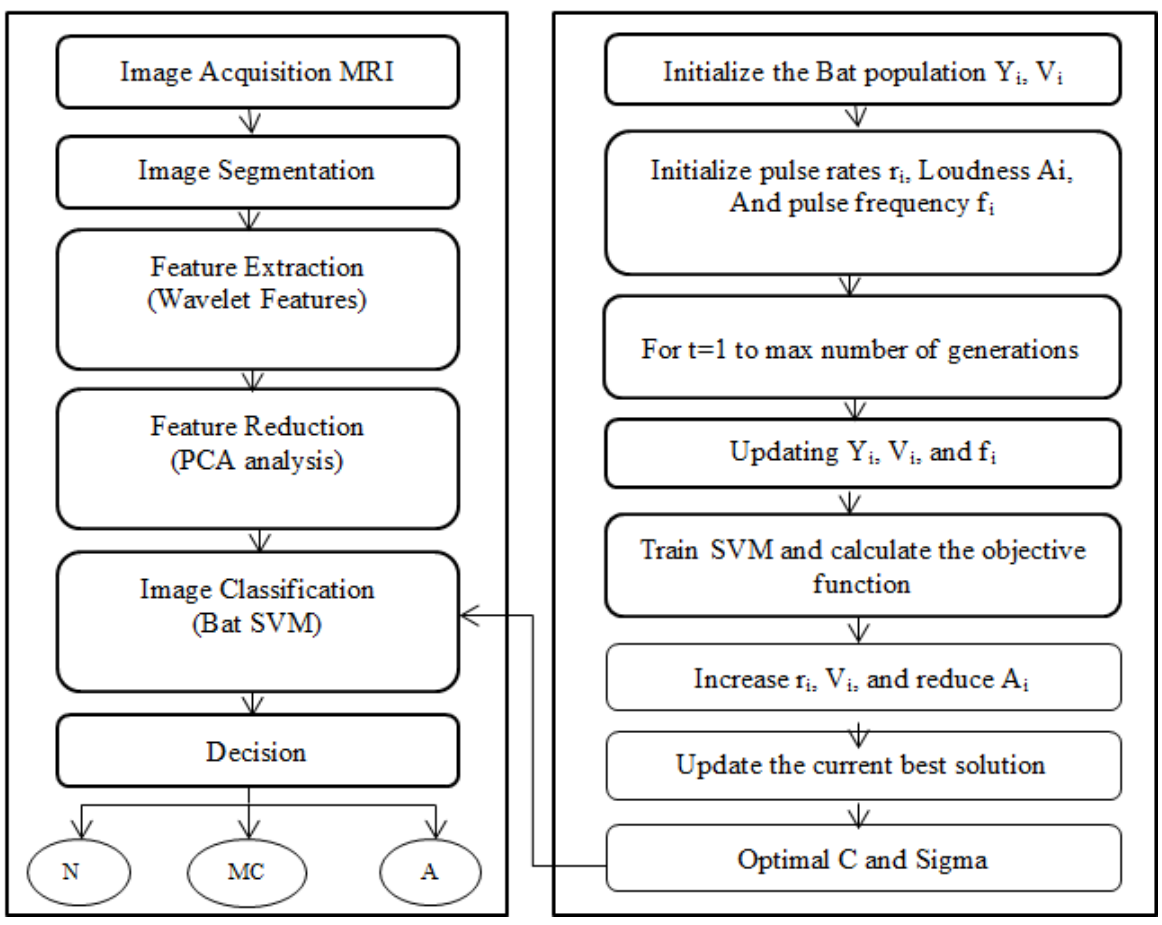

Figure 1. Framework of the proposed model

\subsection{Classification phase}

In the third phase, the most significant reduced features were submitted to machine learning classification technique. SVM classifier is one of the most popular pattern classification approaches to diagnose AD [18-20]. SVM is supervised classification technique based on machine learning theory that improves detection and differentiation of Alzheimer's disease classification techniques. SVMs have important advantages, the accuracy is very high and also a small number of training samples does need to avoid occurring over fitting rather than artificial neural network, decision tree, and Bayesian network. Moreover, we decided in this paper to use the radial basis function (RBF) kernel to get the better performance.

Hence, an optimization algorithm was used to optimize kernel function parameters $\mathrm{C}$ and sigma automatically. The regularization constant $\mathrm{C}$ determines the trade-off between the minimization of fitting error and the maximization of classification margin. Bandwidth (sigma) of the RBF kernel, affect the mapping transformation of data space and alter the complexity degree of sample distribution in the higher dimensional feature space $[21,22]$.

\subsection{Bat-SVM phase}

In our work we used a Bat optimizer in order to make a new Bat-SVM model for SVM parameter optimization problem. In the proposed Bat-SVM model, the SVM parameter values are dynamically improved to maximize the classification accuracy. Furthermore, the classification task of the SVM model performs the obtained optimal parameter values.

Metaheuristic algorithms including evolutionary and swarm intelligence algorithms are now becoming powerful methods for solving many tough problems. Bat algorithm is a recent metaheuristic algorithm introduced by Yang in [23]. It was proposed by modeling the navigating and tracking capability of bats. Bats are fascinating animals. They are the only mammals with wings and they also have advanced capability of echolocation. Bats fly randomly with velocity $\mathrm{Vi}$ at position $\mathrm{Xi}$ with a fixed frequency fm in, varying wavelength $\lambda$ and loudness A to search for prey. They can automatically adjust the wavelength (or 
frequency) of their emitted pulses and adjust the rate of pulse emission $r$ in the range of $[0,1]$, depending on the proximity of their target. In simulations, we have to define the rules how their positions xi and velocities $\mathrm{Vi}$ in a dimensional search space are updated. The new solutions $\mathrm{Xi}(\mathrm{t})$ and velocities $\mathrm{Vi}(\mathrm{t})$ at time $\mathrm{t}$ are given by $[24,25]$. Where, $\beta$ is a random vector generated by a uniform distribution belonging to the closed interval $[0,1]$. The additional control parameters fmin and fmax were set to 0 and 2.0 , respectively. Here $X^{\prime}$ is the current global best solution which is located after comparing all the solutions among all the N bats. See (1)-(3),

$$
\begin{aligned}
& f_{i}=f_{\text {min }}+\left(f_{\text {max }}-f_{\text {min }}\right) \beta \\
& V_{i}(t)=V_{i}(t-1)+\left(X_{i}(t-1)-X^{\prime}\right) f_{i} \\
& X_{i}(t)=X_{i}(t-1)+V_{i}(t)
\end{aligned}
$$

\section{EXPERIMENTAL RESULTS AND DISCUSSION}

In this research, the implemented experiments have been conducted using the MATLAB platform. The experiments were implemented to assess the performance of the proposed model, with variant population size from 10 to 50 that has been used for training and testing. The participant's cases of MCI patients were Males age range 57-60 years, Female age range 50-59 years, and of AD patient's males and females age range $60-70$ years.

\subsection{Dataset}

The dataset that has been used for training and testing the proposed system was obtained from the Radiopaedia.org. It is a free educational radiology resource and it is a one of the highest collections web of radiology cases and reference articles (http://radiopaedia.org/). Hence, we select from the Radiopaedia database. The goal of Radiopaedia to test whether serial magnetic resonance imaging (MRI), positron emission tomography (PET), other biological markers, and clinical and neuropsychological assessment can be combined to measure the progression of mild cognitive impairment (MCI) and early Alzheimer's disease (AD). The data set divided into three previously described classes; NC, MCI and AD the three classes were analyzed the two locations: the right hippocampus (RH) and left hippocampus (LH) these three classes predict Alzheimer case. The participant's cases of MCI patients were Males age range 57-60 years, female age range 50-59 years and of $\mathrm{AD}$ patient's males and females age range 60-70 years.

\subsection{Parameters for SVM and bat algorithm}

The RBF kernel was acquired with the value of $\mathrm{C}$ and sigma parameters are exponentially varied $(C=2 \mathrm{e}-6: 2 \mathrm{e} 14$; sigma=2e-15:2e10), to analyze the general performance of SVM, in order to determine the parameters analysis of SVM. The tuning of the parameters for the optimization algorithms, and setting the parameter for the Bat algorithm have shown to have significant importance; as it improves the performance. The main parameters of Bat algorithm are: Firstly the population size from 10:50. Selection of population size affects the efficiency of the algorithm; therefore it is considered as a very critical issue. The second parameter is the number of generation and takes the value of 1 and 10 . The initial values of pulse rate and loudness parameters were set to 0.5 , and dimension of the search variables $D=2$. The minimum frequency equal 0 and maximum frequency equal 2. The output of segmentation phase for four samples of the data set was demonstrated in Figure 2 from segmented MRI biomedical image 1 to segmented MRI biomedical image 4, it shows the most disease areas which is hippocampus in the four samples of the data set.

Figure 3 illustrates the features extraction parameters results of eleven MRI biomedical images named 1-F to 11-F for left, right hippocampus size and the corresponding average size 13 statistical features that extracted using the 2-D wavelet transform to build feature vector by the brain (contrast, correlation, energy, homogeneity, mean, standard deviation, entropy, RMS, variance, smoothness, kurtosis, skewness, IDM, sze of $\mathrm{mmL}$, size of $\mathrm{mmR}$, and region size mean). The 13 statistical features that extracted using the 2-D wavelet transform are all important to build feature vector by the brain, but the most significant features that affected on the next stage of the proposed model to classify and diagnose the AD disease is hippocampus region size in the structural MRI images. Since, early memory decline is correlated to the appearance of reductions in hippocampus volume that is sensitive to the first stage of AD. Then in the proposed model wavelet transform is used to extract images features then PCA is applied to minimize the features dimensions then the reduced features were used. The third phase of the proposed model, classification phase depends on the most significant reduced features for submission to machine learning classification technique SVM classifier which is one of the most popular pattern classification approaches to diagnose AD. Furthermore, in this work a new Bat-SVM model based on Bat optimizer to optimize the SVM parameter. 

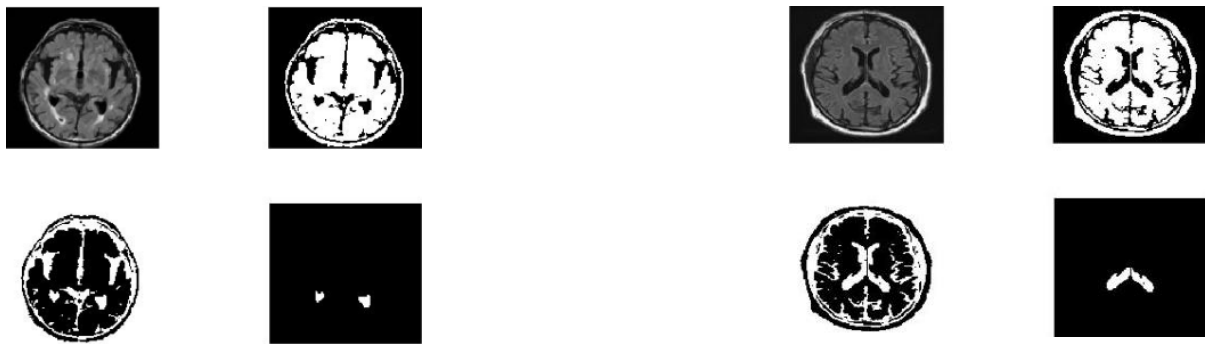

(a)

(b)
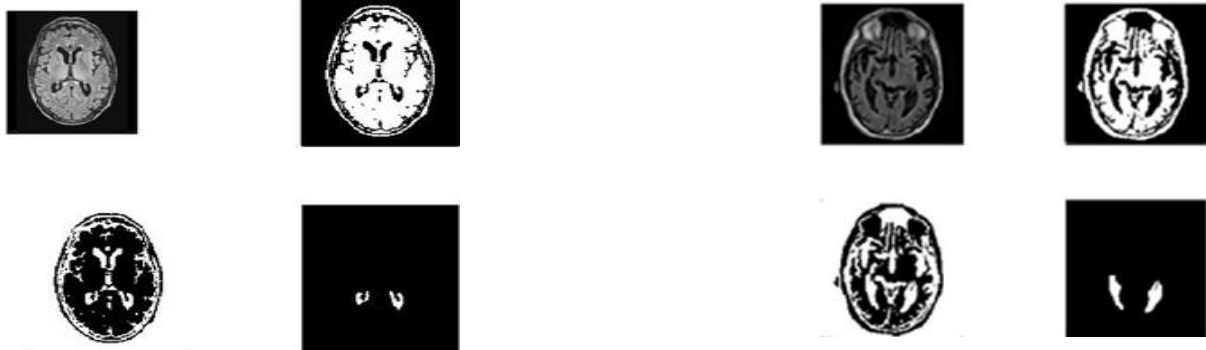

(c)

(d)

Figure 2. Segmentation MRI biomedical, (a) Image 1, (b) Image 2, (c) Image 3, (d) Image 4

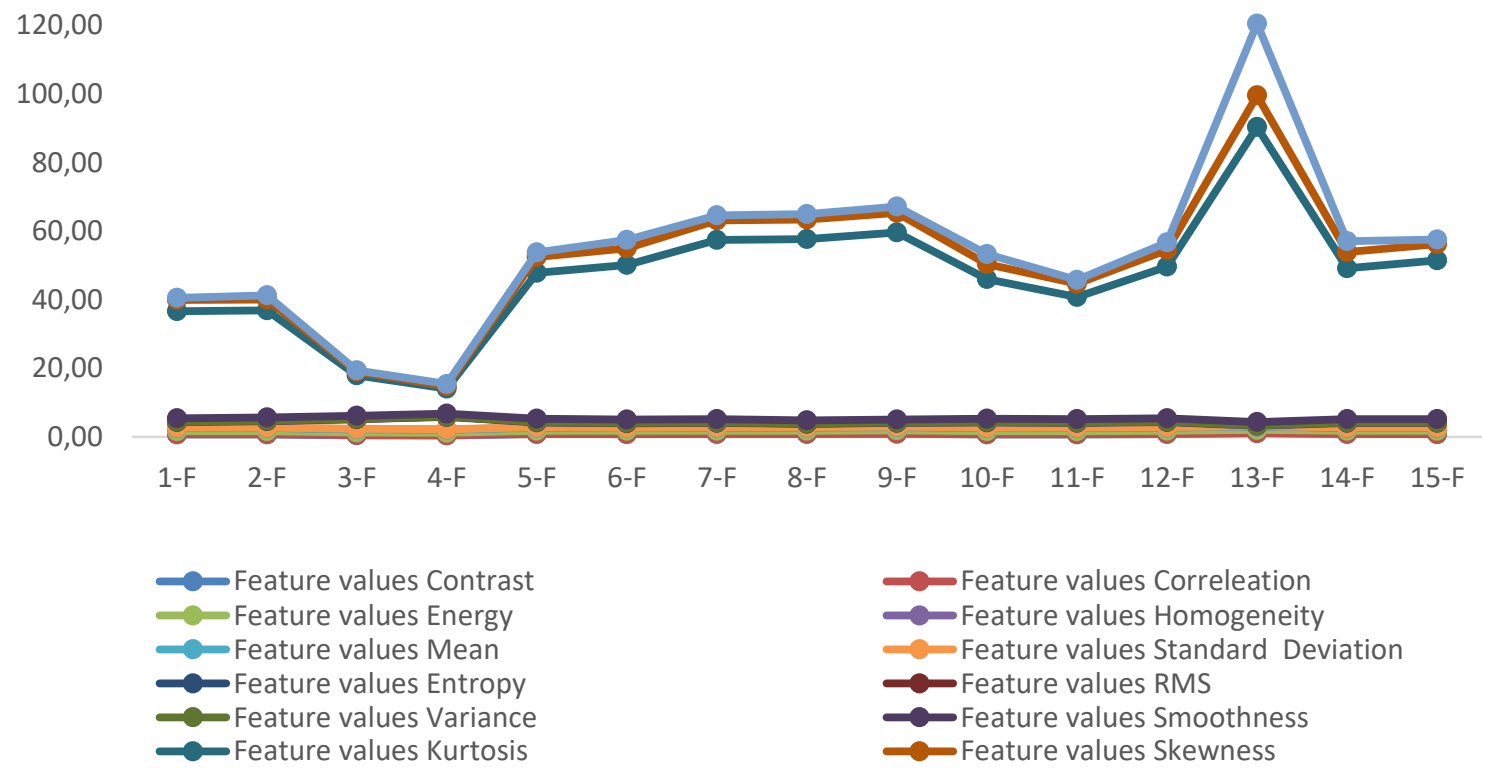

Figure 3. Feature extraction parameters results

\subsection{Classification based on SVM}

For evaluating the performance of classification without optimize any SVM parameters, HoldOut and 7-fold cross validation were used and the accuracy was calculated three times for each one using different types of Kernel as illustrated in Table 1. It is concluded that from the experimental results the maximum accuracy for using HoldOut is equal to $64 \%$ but for 7 -fold the highest accuracy is reached to $82 \%$ this is means the accuracy of 7-fold is larger than HoldOut. 


\begin{tabular}{ccccc}
\multicolumn{5}{c}{ Table 1. Classification accuracy using SVM } \\
\hline \multirow{2}{*}{ Cross validation } & $\begin{array}{c}\text { Accuracy of } \\
\text { linear kernel }\end{array}$ & $\begin{array}{c}\text { Accuracy of RBF } \\
\text { kernel }\end{array}$ & $\begin{array}{c}\text { Accuracy of } \\
\text { polynomial kernel }\end{array}$ & $\begin{array}{c}\text { Accuracy of } \\
\text { quadratic kernel }\end{array}$ \\
\hline \multirow{3}{*}{ HoldOut } & $64 \%$, & $58 \%$, & $55 \%$, & $56 \%$, \\
& $63 \%$, & $59.6 \%$ & $50 \%$, & $53 \%$, \\
\multirow{3}{*}{ 7-fold } & $63.8 \%$ & $54.4 \%$ & $54 \%$ & $51 \%$ \\
& $60.36 \%$ & $53.02 \%$, & $46.98 \%$, & $31.90 \%$ \\
& $77.98 \%$ & $60.20 \%$, & $52.65 \%$, & $39.29 \%$ \\
& $82.03 \%$ & $56.03 \%$ & $56.35 \%$ & $52.22 \%$ \\
\hline
\end{tabular}

\subsection{Classification based on Bat-SVM}

For classification performance evaluation with optimize the parameters of SVM using Bat optimizer, 7-fold cross validation is used then the classification accuracy is calculated to evaluate classifier performance and the corresponding optimized RBF Kernel parameters are summarized in Tables 2 and 3 based on the different number of generation and different population size of bats moreover, the corresponding running time is calculated. Experimental results of classification based on Bat-SVM gives better values of accuracy than the classification without optimize the parameters and the classification accuracy reached to $95.36 \%$. It was established that there is a direct relation between the running time and the population size; as the running time of the proposed Bat-SVM model increases with the population size as shown in Figure 4. At the same time, the accuracy is increased with the number of generation as shown in Figure 5.

Table 2. Classification accuracy using Bat-SVM, number of generation=1

\begin{tabular}{ccccc}
\hline Population size N & Optimal sigma & Optimal c & Accuracy & Running time (second) \\
\hline 10 & 0.0291 & 6.6253 & $91.39 \%$ & 92.14 \\
20 & 0.0092 & 2.3192 & $90.10 \%$ & 173.98 \\
30 & 0.0015 & 1.0851 & $93.32 \%$ & 263.10 \\
40 & 0.0041 & 1.5099 & $88.69 \%$ & 350.01 \\
50 & 0.0011 & 2.3359 & $95.36 \%$ & 485.99 \\
\hline
\end{tabular}

Table 3. Classification accuracy using Bat-SVM, number of generation=10

\begin{tabular}{ccccc}
\hline Population size N & Optimal sigma & Optimal c & Accuracy & Running time (second) \\
\hline 10 & 0.0047 & 2.2986 & $95.36 \%$ & 482.70 \\
20 & 0.0213 & 4.4667 & $92.5 \%$ & 951.38 \\
30 & 0.0129 & 5.4794 & $93.31 \%$ & 1639.48 \\
40 & 0.0068 & 1.8441 & $90.75 \%$ & 1891.81 \\
50 & 0.0041 & 1.8648 & $95.56 \%$ & 2876.14 \\
\hline
\end{tabular}

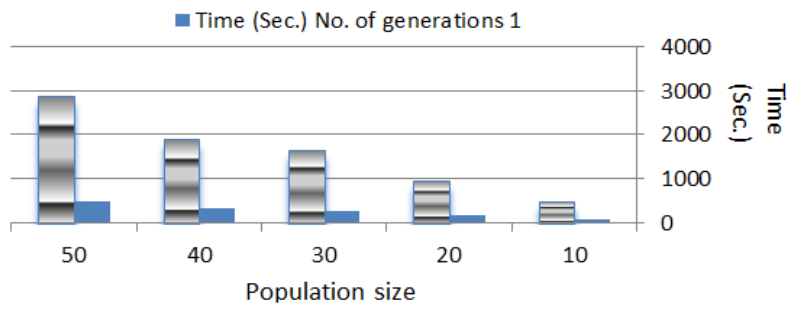

Figure 4. Effect of population size on the running time of the model Bat-SVM

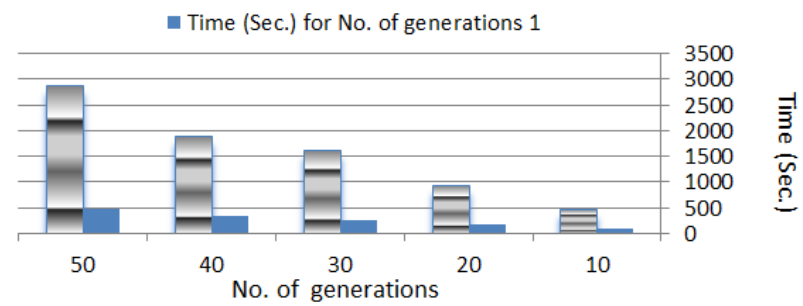

Figure 5. Effect the accuracy of the Bat-SVM model with number of generation 


\section{CONCLUSION}

In this paper a new computer aided diagnosis model was proposed to assist in the detection of the Alzheimer's disease in early stage. In the proposed model, Bat algorithm for the first time is used as optimizer for RBF kernel parameters. We tested the proposed method on different datasets and compared it with the classification without using optimizer. The experimental results classification based on Bat-SVM achieved higher accuracy than the classification without implement optimization step and the accuracy reached to $95.36 \%$. The Bat optimizer has the ability for optimize SVM parameters to improve the accuracy rate and the proposed model can yield promising results.

\section{REFERENCES}

[1] T. William and B. Laura, "Alzheimer's disease facts and figures," Alzheimer's Association: Alzheimer's \& Dementia, vol. 9, no. 2, pp. 208-245, 2013.

[2] M. L. Ries, C. M. Carlsson, H. A. Rowley, M. A. Sager, C. E. Gleason, S. Asthana, and S.C. Johnson, "Magnetic resonance imaging characterization of brain structure and function in mild cognitive impairment: A review," Journal of the American Geriatrics Society, vol. 56, no. 5, pp. 920-934, 2008.

[3] C. Davatzikos, Y. Fan, X. Wu, D. Shen, and S.M. Resnick, "Detection of prodromal Alzheimer's disease via pattern classification of magnetic resonance imaging," Neurobiology of Aging, vol. 29, no. 4, pp. 514-523, 2008.

[4] R. Cuingnet, E. Gerardin, J. Tessieras, G. Auzias, S. Lehricy, M. O. Habert, M. Chupin, H. Benali, and O. Colliot, "Automatic classification of patients with Alzheimer's disease from structural MRI: A comparison of ten methods using the ADNI database," NeuroImage, vol. 56, no. 2, pp. 766-781, 2011.

[5] N. Villain, B. Desgranges, F. Viader, V. De La Sayette, F. Mézenge, B. Landeau, J. C. Baron, F. Eustache, and G. Chételat, "Relationships between hippocampal atrophy, white matter disruption, and gray matter hypometabolism in Alzheimer's disease," Journal of Neuroscience : the official journal of the Society for Neuroscience, vol. 28, no. 24, pp. 6174-6181, 2008.

[6] M. Chupin, E. G'erardin, R. Cuingnet, C. Boutet, L. Lemieux, S. Lehéricy, H. Benali, L. Garnero, and O. Colliot, "Fully automatic hippocampus segmentation and classification in Alzheimer's disease and mild cognitive impairment applied on data from ADNI," Hippocampus, vol. 19, no. 6, pp. 579-587, 2009.

[7] E. Gerardin, G. Chételat, M. Chupin, R. Cuingnet, B. Desgranges, H. S. Kim, and F. Eustache, "Multidimensional classification of hippocampal shape features discriminates Alzheimer's disease and mild cognitive impairment from normal aging," NeuroImage, vol. 47, no. 4, pp. 1476-1486, 2009.

[8] B. Gutman, Y. Wang, J. Morra, A. W. Toga, and P. M. Thompson, "Disease classification with hippocampal shape invariants," Hippocampus, vol. 19, no. 6, pp. 572-578, 2009.

[9] G. Gainotti, A. Acciarri, A. Bizzarro, C. Marra, C. Masullo, S. Misciagna, T. Tartaglione, A. Valenza, and C. Colosimo, "The role of brain infarcts and hippocampal atrophy in subcortical ischaemic vascular dementia," Neurological Sciences: Official journal of the Italian Neurological Society and of the Italian Society of Clinical Neurophysiology, vol. 25, pp. 192-197, 2004.

[10] F. Falahati, E. Westman, and A. Simmons, "Multivariate data analysis and machine learning in Alzheimer's disease with a focus on structural magnetic resonance imaging," Journal of Alzheimer's Disease: JAD, vol. 41, no. 3, pp. 685-08, 2014

[11] J. Ye, K. Chen, T. Wu, J. Li, Z. Zhao, R. Patel, M. Bae, R. Janardan, H. Liu, G. Alexander, and E. Reiman, "Heterogeneous data fusion for alzheimer's disease study," In Proceedings of the 14th ACM SIGKDD International Conference on Knowledge Discovery and Data Mining, pp. 1025-1033, 2008.

[12] A. Olfa, M. Maxim, B. Jenny, A. Michèle, Gwénaëlle Catheline, and Chokri Ben Amar, “Alzheimer's disease diagnosis on structural MR images using circularharmonic functions descriptors on hippocampus and posteriorcingulate cortex," Computerized Medical Imaging and Graphics, vol. 44, pp. 13-25, 2015.

[13] M. Sezgin and B. Sankur, "Survey over image thresholding techniques and quantitative performance evaluation," Journal of Electronic Imaging, vol. 13, no. 1, pp. 146-165, 2004.

[14] M. E. Tagluk, M. Akin, and N. Sezgin, "Classification of sleep apnea by using wavelet transform and artificial neural networks," Expert Systems with Applications, vol. 37, no. 2, pp. 1600-1607, 2010.

[15] J. Camacho, J. Pic'o, and A. Ferrer, "Corrigendum to The best approaches in the on-line monitoring of batch processes based on PCA: Does the modelling structure matter," Analytica chimica acta, vol. 642, no. 1-2, pp. 59-68, 2010.

[16] I. Jolliffe, "Principal components in regression analysis," In Principal component analysis, pp. 129-155, Springer, New York, NY, 1986.

[17] López M, Ramírez J, Górriz J. M, Álvarez I, Salas-Gonzalez D, Segovia F, and Chaves R, "SVM-based CAD system for early detection of the Alzheimer's disease using kernel PCA and LDA," Neuroscience Letters, vol. 464, no. 3, pp. 233-238, 2009.

[18] B. Magnin, L. Mesrob, S. Kinkingnéhun, M. Pélégrini-Issac, O. Colliot, M. Sarazin, and H. Benali, "Support vector machines-based classification of Alzheimer disease from whole-brain anatomical MRI," Neuroradiology; vol. 51, pp.73-83, 2009.

[19] Agus Eko Minarno, Fauzi Dwi Setiawan Sumadi, Hardianto Wibowo, and Yuda Munarko, "Classification of batik patterns using K-Nearest neighbor and support vector machine," Bulletin of Electrical Engineering and Informatics vol. 9, no. 3, pp. 1260-1267, June 2020. 
[20] S. A. Taie and W. Ghonaim, "CSO-based algorithm with support vector machine for brain tumor's disease diagnosis," IEEE International Workshop on Behavioral Implications of Contextual Analytics (PerCom Workshops), pp. 183-187, 2017.

[21] F. F. Wang and Y. R. Zhang, "The support vector machine for dielectric target detection through a wall," Progress In Electromagnetics Research Letters, vol. 23, pp. 119-128, 2011.

[22] Li D, Yang W, and Wang S, "Classication of foreign bers in cotton lint using machine vision and multi-class support vector machine," Computers and Electronics in Agriculture, vol. 4, no. 2, pp. 274-279, 2010.

[23] Yang X. S., "A new meta heuristic bat-inspired Algorithm," In Nature inspired cooperative strategies for optimization (NICSO 2010), vol. 284, pp. 65-74, 2010.

[24] Yang, X. S., and He, X, "Bat algorithm: literature review and applications," International Journal of Bio-inspired computation, vol. 5, no. 3, pp. 141-149, 2013.

[25] Wafaa Ghonaim and Shereen A. Taie, "A new image segmentation algorithm based on particle swarm optimization and rough set," Journal of Computers, vol. 13, no. 1, pp. 130-138, Jan 2018.

\section{BIOGRAPHIES OF AUTHORS}
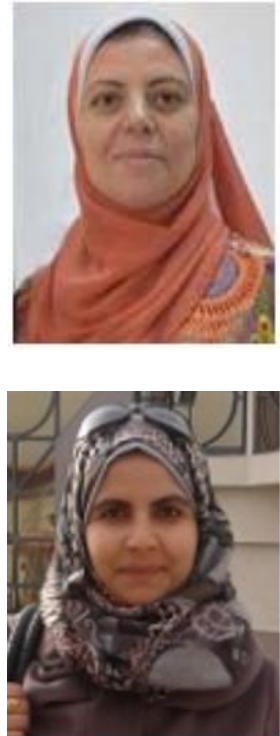

Shereen A. Taie is an associate professor in Computer Science Department, Faculty of Computers and Information, Fayoum University, Egypt and Head of Center of Electronic Courses Production, Fayoum University, Egypt. Her current research interest is in the area of Machine Learning, Image Processing, and Computer Vision.

Wafaa Ghonaim is working as a lecture at faculty of Science, Al-Azhar University. Her current research interest is in the area of computational intelligence based on rough set theory, swarm optimization, image processing, and image retrival. 